\title{
Dezentralisierung ist kein Allheilmittel! - Zur Notwendigkeit einer kontext- spezifischen Dezentralisierungspolitik am Beispiel der Kommunalentwicklung in Südafrika
}

\section{Theo Rauch, Pretoria}

\section{Zur Rolle von Institutionen im Prozess gesellschaftlicher Entwicklung}

Institutionen werden von der zeitgenössischen Entwicklungspolitik gemeinhin als der entscheidende Hebel zur Entwicklung angesehen. Nicht mehr der Anstoss zu technischen Innovationen, sondern die Förderung bestimmter Demokratieformen, von Privatisierung, von Dezentralisierung oder von Organisationsentwicklung im Allgemeinen stehen im Vordergrund der Interventionen aller Entwicklungsorganisationen, und die Entwicklung der institutionellen Kapazitäten gilt dabei als der zentrale Ansatzpunkt.

In den Gesellschaftswissenschaften bestimmt die Neue Institutionenökonomie (NIÖ) den «Main Stream» des Denkens und Forschens. Ihr kommt das Verdienst zu, die Aufmerksamkeit der Ökonomen stärker auf institutionelle Aspekte, auf Anreizsysteme, Regulierungsmechanismen und Normen gelenkt zu haben. Das Motto: «Get the Institutions Right» hat das allzu reduktionistische neoklassische Motto «Get the Prices Right» ergänzt oder gar ersetzt. Diese neue Aufmerksamkeit für institutionelle Aspekte, für Regulierungsformen und ihre Wirkungen auf wirtschaftliche und gesellschaftliche Entwicklungen ist aus entwicklungstheoretischer wie aus entwicklungspraktischer Sicht zu begrüßen. Entwicklungsbemühungen, die die institutionelle Dimension ausblenden, sind - das belegen vielfältige Erfahrungen - zum Scheitern verurteilt.

Die Auseinandersetzung um die Rolle von Institutionen im Entwicklungsprozess bezieht sich also nicht auf die Frage der Wichtigkeit der institutionellen Dimension. Diese ist unumstritten. Sie bezieht sich auf die Art des Verhältnisses zwischen der institutionellen Sphäre und der ökonomischen und gesellschaftlichen Sphäre oder, plakativ verkürzt, auf die Beziehung zwischen Institutionen und Entwicklung.

Die NIÖ und der «Main Stream» der entwicklungspolitischen Praxis betrachtet einseitig die Wirkung von Institutionen auf den Entwicklungsprozess, sieht die institutionelle Sphäre als von gesellschaftlichen Entwicklungsprozessen weitgehend unabhängige, ökonomische und gesellschaftliche Entwicklungsprozesse als weitgehend davon abhängige Variable. Der
NIÖ (vgl. NoRTH 1992) geht es primär darum, den Einfluss von institutionellen Regulierungs-, Anreiz- bzw. Normensystemen auf wirtschaftliches Verhalten und damit auf Entwicklung zu analysieren. In der Entwicklungspolitik werden dementsprechend institutionelle Reformen als Ansatzpunkt oder als Instrumentvariable und die dadurch zu bewirkenden Verbesserungen von Wirtschaftsleistung und Lebensbedingungen als Zielvariable gesehen. Die NIÖ sucht nach generellen, allgemeingültigen Aussagen dazu, wie «richtige», d.h. entwicklungsförderliche Institutionen gestaltet sein müssen. (Nach dem Scheitern der «großen Theorien» wagt man sich offenbar wieder an die Formulierung allgemeiner Entwicklungstheorien!). Die meisten entwicklungspolitischen Organisationen postulieren, mit Mehr-Parteien-Demokratie, Dezentralisierung politischer und bürokratischer Macht und Privatisierung solch global gültige Antworten bereits gefunden $\mathrm{zu}$ haben.

Die Frage nach den Einflussfaktoren auf die tatsächliche Ausgestaltung institutioneller Systeme, d.h. die Frage, wie die ökonomische und gesellschaftliche Sphäre auf die institutionelle Sphäre wirkt, bleibt unbeantwortet. Dass etwa subsistenzbäuerliche, auf starken familiären Beziehungen basierende, ländliche Gesellschaften andere Regulierungsmechanismen oder Demokratie- bzw. Herrschaftsformen hervorbringen und vielleicht auch benötigen als hochindustrialisierte, weitgehend anonyme städtische Industriegesellschaften, bleibt ausgeblendet. Zusammenhänge zwischen Technologieniveau und Art der institutionellen Regulierung werden ebensowenig thematisiert.

Die Ausblendung der Wirkungsrichtung von Entwicklung zu einer ahistorischen Betrachtungsweise von Institutionen führt dazu, dass real existierende institutionelle Systeme nicht im Kontext der jeweiligen Wirtschaftsweise und des Gesellschaftssystems betrachtet, sondern gemessen an vermeintlich allgemeingültigen Erkenntnissen über «richtige» Institutionen werden. Institutionelle Reformen wie Dezentralisierung, Liberalisierung, Privatisierung werden folglich per se, d.h. unabhängig vom jeweiligen sozioökonomischen Kontext, als überlegen und erstrebenswert angesehen.

Im Gegensatz dazu geht dieser Beitrag davon aus, dass die Zusammenhänge zwischen Entwicklung i.S. von Veränderungen in der wirtschaftlichen, technischen und gesellschaftlichen Sphäre - und Insti- 
tutionen wechselseitiger Natur sind. Die Art dieser Zusammenhänge wird durch Abbildung 1 illustriert.

Folgende Zusammenhänge zwischen sozioökonomischer und institutioneller Entwicklung lassen sich demnach unterscheiden (vgl. RAUCH 1996: 181f.):

(I) Institutionelle Entwicklung als Resultat wirtschaftlicher und gesellschaftlicher Entwicklung

(1) Institutionelle Entwicklung als Antwort auf einen «objektiven» Regulierungsbedarf: Der Prozess der materiellen Produktionsentwicklung ist verbunden mit zunehmender Arbeitsteilung und Spezialisierung und damit auch mit zunehmender Differenzierung und intensivierter Integration verschiedener Wirtschaftseinheiten (vgl. Elias 1976: 312ff.). Daraus resultiert ein zunehmender Regulierungsbedarf, also eine zunehmende Notwendigkeit der Institutionalisierung der Gesellschaft. Was z. B. in der Subsistenzwirtschaft noch durch einfache internalisierte Werthaltungen (vermittelt z. B. über die Institution der Initiationsriten) geregelt werden konnte (HYDEN 1987), bedarf nun formalisierter Gesetze.

(2) Institutionelle Entwicklung als Resultat gesellschaftlicher Interessenkonstellationen: $\mathrm{Da}$ institutionalisierte Regulierungen in ihrer Wirkung auf verschiedene gesellschaftliche Gruppen nicht neutral sind, sondern die Chancenverteilung in einer Gesellschaft entscheidend beeinflussen können,

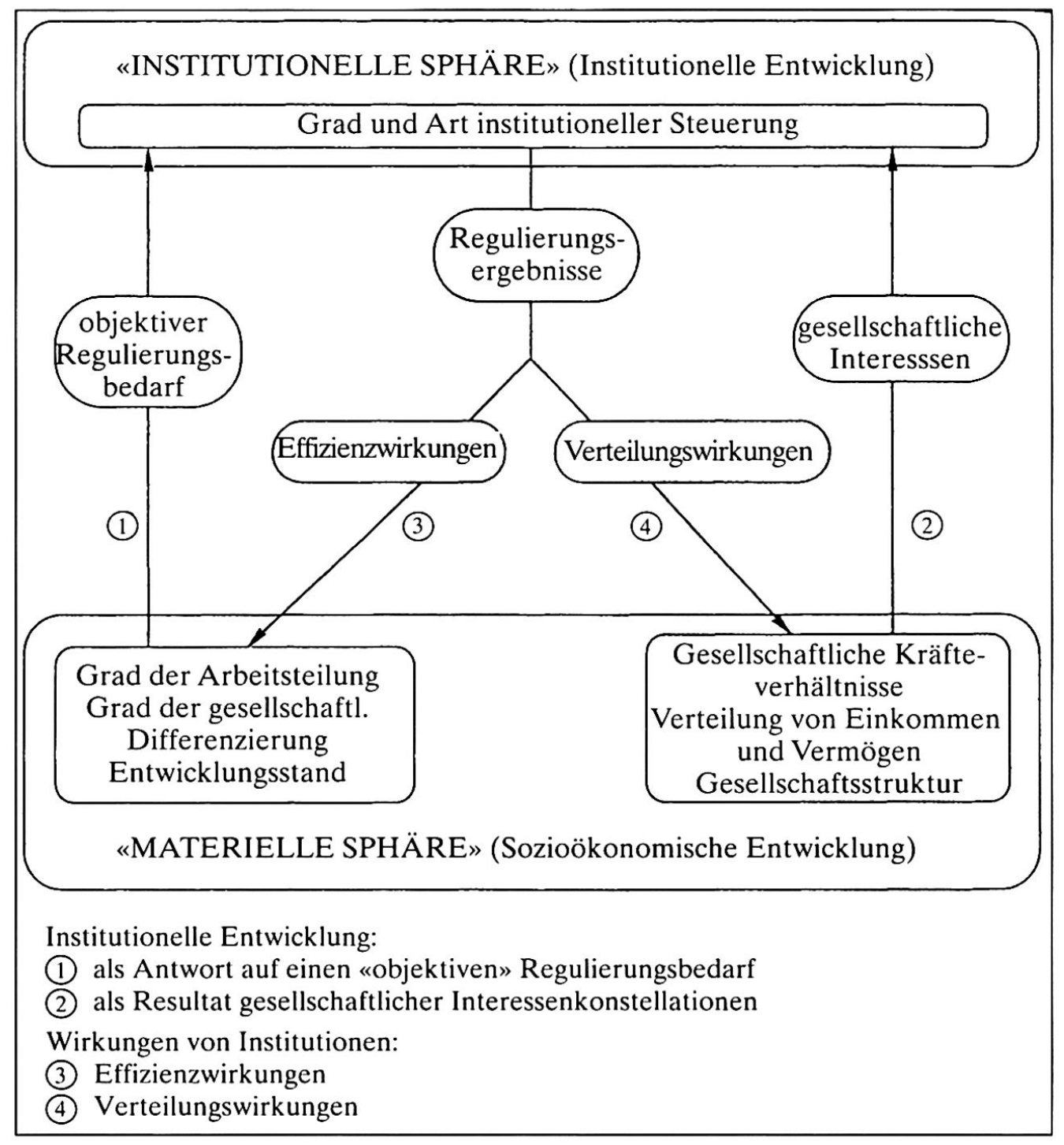

Abb. 1: Die Interdependenzen zwischen «materieller» und «institutioneller Sphäre» im Entwicklungsprozess Interrelations between the socio-economic and institutional dimensions of development Les interdépendances entre les sphères «matérielle» et «institutionnelle» dans le processus de développement Entwurf: T. RAUCH 
werden die verschiedenen gesellschaftlichen Gruppen versuchen, die Bildung von Institutionen in ihrem Sinn zu beeinflussen. Art und Grad der Regulierung sind folglich nicht allein erklärbar aus dem objektiven Regulierungsbedarf, sondern auch aus den dominierenden gesellschaftlichen Interessen. Wo gesellschaftlich mächtige Gruppen Nutzen aus verstärkter Regulierung ziehen können, kann der Grad der Institutionalisierung auch weit über den objektiven Regulierungsbedarf hinaus gehen (etwa im Fall der übermässigen Aufblähung des Staatsapparates in Entwicklungsländern). Wo solche Gruppen hingegen kein Interesse an Regulierung haben, kann der Grad an Institutionalisierung auch hinter den objektiven Notwendigkeiten zurückbleiben (etwa im Fall des staatlich unkontrollierten Faustrechtes und Freibeutertums bei der Nutzung der Amazonaswälder in Brasilien).

(II) Wirtschaftliche und gesellschaftliche Entwicklung als Resultat institutioneller Regulierung

(3) Die Effizienzwirkungen von Institutionen:Institutionelle Steuerung wirkt auf die sozio-ökonomische Entwicklung einer Gesellschaft zurück. So kann z.B. eine angemessene rechtliche Regulierung von Geschäftsbeziehungen, welche individueller Initiative einen angemessenen Freiraum gibt, sie aber daran hindert, den Freiraum anderer Individuen einzuschränken, das nötige Vertrauen für Investitionen schaffen und so Kräfte für wirtschaftliche Entwicklung freisetzen. Der Zusammenbruch der zentralverwaltungswirtschaftlichen Systeme zeigt andererseits, wie unangemessene Regulierungsformen sozio-ökonomische Entwicklung behindern können.

(4) Die Verteilungswirkungen von Institutionen: Die Art der institutionellen Steuerung bleibt nicht ohne Konsequenzen für die gesellschaftlichen Kräfteverhältnisse und für die Verteilung von Ressourcen und Einkommen. Bürokratische Steuerung mag anderen gesellschaftlichen Gruppen zugute kommen als eine Steuerung durch den Marktmechanismus. Kommunale Regelung von Nutzungsrechten an Grund und Boden hat andere Verteilungswirkungen als Privateigentum.

\section{Dezentralisierung als Reform des politisch- institutionellen Systems}

Dezentralisierung bedeutet Verlagerung von Entscheidungsmacht, von Verantwortung und von Kontrolle finanzieller und personeller Ressourcen auf untergeordnete, bürgernähere staatliche Ebenen bzw. eigenständige Organisationseinheiten oder Gebietskörperschaften. Es handelt sich also um eine Reform eines politisch-institutionellen Systems.

Dezentralisierung impliziert somit auch Umverteilung von Macht, d.h. Machtverlust zentralstaatlicher Instanzen. Dezentralere Formen der Regulierung müssen folglich von bestimmten gesellschaftlichen Gruppen gegen andere Gruppen durchgesetzt werden, und ihre reale Umsetzung muss von diesen Gruppen gestaltet und getragen werden. Es bedarf dezentraler Akteure, welche mehr Macht wollen und handhaben können. Die Durchsetzbarkeit und Machbarkeit von Dezentralisierung hängt also von gesellschaftlichen Bedingungen, von Einflussfaktoren außerhalb des politischinstitutionellen Systems ab.

Dezentralisierung ist kein Selbstzweck. Dezentrale Regulierung ist nicht per se besser als zentrale Steuerung. Der Vorteil der Dezentralisierung ist an dem konkreten Nutzen zu messen, den sie für die Bevölkerung bzw. für die verschiedenen Bevölkerungsgruppen des betreffenden Staates bringt, d.h. sie ist an ihrer Entwicklungswirkung zu messen. Eine Dezentralisierung, die zu erhöhter Korruption, Ineffizienz, Bereicherung lokaler Eliten und Massenverarmung führt, ist nicht unterstützungswürdig. Dezentralisierung ist ein Instrument, welches je nach dem jeweiligen historischen Kontext sozio-ökonomische Entwicklung fördern oder auch hemmen kann.

Es gilt also bei einer Dezentralisierungsförderungspolitik die Frage nach Durchsetzbarkeit und Zweckmäßigkeit von dezentraleren Regulierungsformen im Kontext der jeweiligen gesellschaftlichen Bedingungen und Ziele zu betrachten (vgl. Abb. 2).

\section{Gesellschaftliche Faktoren für die Durchsetz- barkeit dezentraler Regulierung}

Es gibt eine Reihe wichtiger Einflussfaktoren auf den Erfolg einer Dezentralisierungspolitik, welche dem institutionellen System immanent und welche machbar bzw. gestaltbar sind. Hierzu gehören

- die Formulierung klarer rechtlicher und politischer Grundlagen für den Reformprozess,

- die Benennung starker, möglichst interministerieller Träger für die Steuerung und Koordination des Dezentralisierungsprozesses,

- die Erarbeitung eines Dezentralisierungskonzeptes, welches die angestrebte Verteilung von Aufgaben und Verantwortlichkeiten sowie die Formen von Koordination und (wechselseitiger) Kontrolle zwischen den verschiedenen staatlichen Ebenen klärt, - die Verabschiedung eines detaillierten, phasen- 


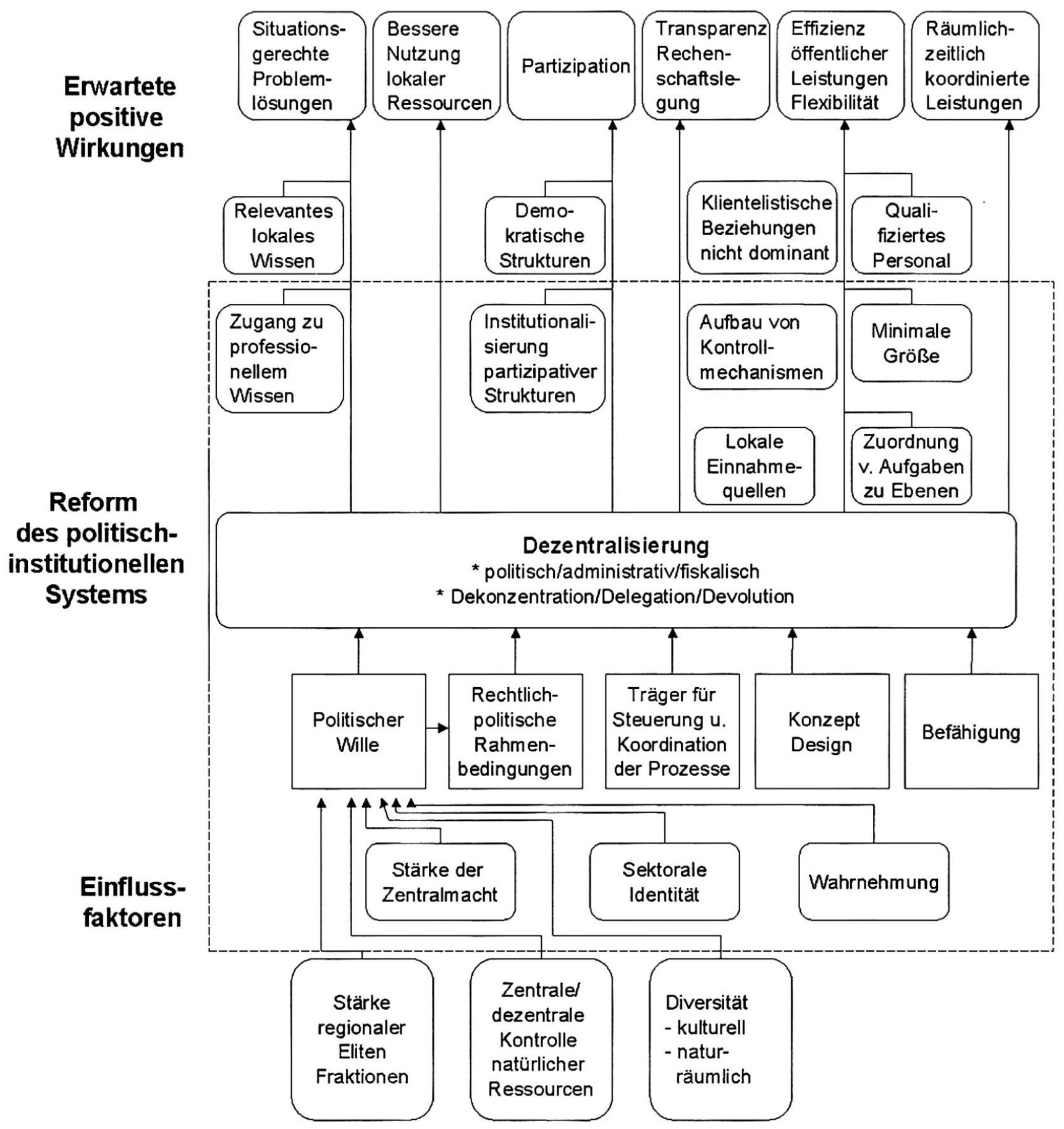

Abb. 2: Modell der Einflussfaktoren und Ziele der Dezentralisierung

Factors and objectives of decentralisation

Les facteurs et les objectifs de la décentralisation

Entwurf: T. RAUCH 


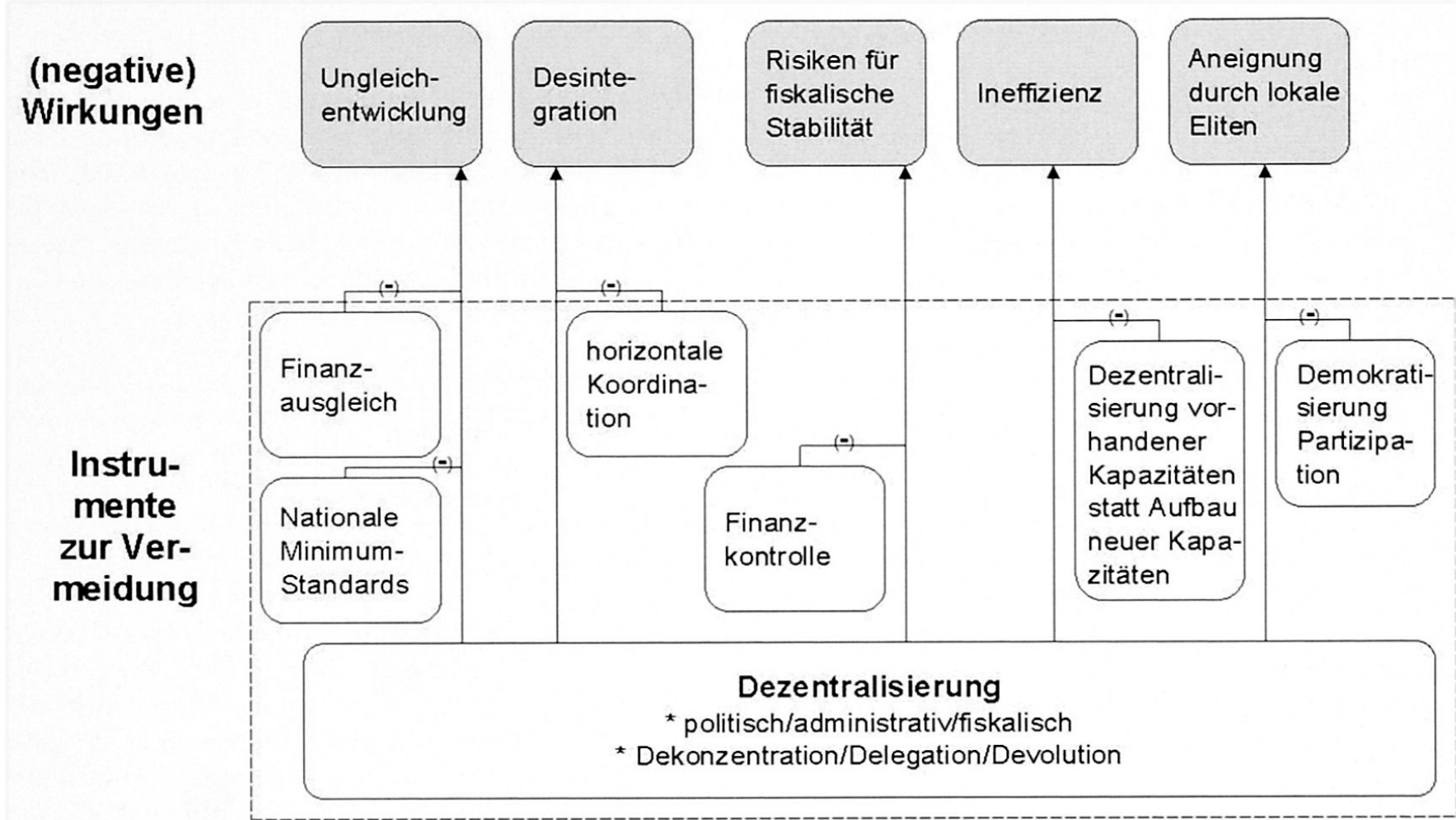

Abb. 2a: Unerwünschte Wirkungen von Dezentralisierung und Instrumente zu deren Begrenzung

Possible negative effects of decentralisation and mechanisms to minimize them

Les effets non désirés de la décentralisation et les instruments pour les limiter

Entwurf: T. RAUCH

weisen Umsetzungsprogramms, in welchem die einzelnen administrativen und rechtlichen Schritte für die Übergabe von Finanzen, Personal und Ausrüstungsgegenständen für alle Beteiligten verbindlich geregelt sind,

- Maßnahmen zur Befähigung dezentraler Gebietskörperschaften zur Übernahme der neuen Aufgaben.

In den meisten Ländern der sog. Dritten Welt, in denen offiziell eine Dezentralisierungspolitik verfolgt wird, gibt es entsprechende rechtliche und politische Grundlagen und es gibt ausländische Organisationen der Entwicklungszusammenarbeit, welche die Entwicklung von Konzepten durch das zuständige nationale Ministerium unterstützen und sich um die Befähigung dezentraler Gebietskörperschaften kümmern. Was meist für eine zielstrebige und erfolgreiche Umsetzung fehlt, sind wirklich starke Träger und verbindliche, schlüssige Umsetzungsprogramme. Die zuständigen Ministerien für Gemeindeangelegenheiten (oder Dezentralisierungsministerien) sind gegenüber den etablierten mächtigen Ressorts (Erziehung, Gesundheit, Landwirt- schaft etc.) meist nicht sehr durchsetzungsfähig. Letztere verhalten sich beim Vollzug der konkreten administrativen Umsetzungsschritte oft mehr als hinhaltend.

Die Dezentralisierungsbefürworter beklagen in solchen Fällen den fehlenden politischen Willen. Dieser ist zweifellos der entscheidende Faktor für die Durchsetzbarkeit von Dezentralisierungsprozessen. Ausgehend von der Annahme, dass niemand leicht davon zu überzeugen sein wird, dass er oder sie die Abgabe von Macht und Ressourcenkontrolle wollen soll, wäre es gesellschaftspolitisch naiv, von einem einhelligen politischen Willen zur Dezentralisierung auszugehen. Umverteilung von Macht auf dezentrale Instanzen wird stets ein umstrittener politischer Prozess sein, in welchem jeder einzelne Schritt zwischen Befürwortern und Gegnern umkämpft ist. Wie stark in einem Land der politische Wille zugunsten einer Dezentralisierung ist, hängt teilweise von Einflussfaktoren ab, die Teil des politischen-institutionellen Systems sind, teilweise aber auch von ökonomischen Faktoren und von gesellschaftlichen Kräfteverhältnissen. 
Wichtige dem politisch-institutionellen System zuzuordnende Einflussfaktoren auf Dezentralisierungsbereitschaft sind:

(1) Die Stärke der Zentralregierung: Ist die nationale Regierung leistungsfähig, in ihrer Stärke anerkannt oder militärisch durchsetzungsfähig, werden dezentralisierungsorientierte Fraktionen es schwer haben sich durchzusetzen. Ist hingegen der Zentralstaat bankrott bzw. in der Krise, ist er nicht mehr in der Lage, das Machtmonopol aufrechtzuerhalten und seine Legitimation gegenüber der Bevölkerung zu erhalten, gibt es kaum noch Ressourcen zu kontrollieren und zu verteilen, dann erlahmt tendenziell der Wille, alle Macht und Verantwortung zu zentralisieren. Wenn die Kassen leer sind, liegt es nahe, die Verantwortung zu dezentralisieren (vgl. Rauch 1986: 52f.). Unter solchen Bedingungen ist Dezentralisierung zwar leichter durch setzbar, die Chancen, dass sie zu positiven Ergebnissen führt, sind bei einer schwachen Zentralregierung aber geringer.

(2) Sektorale Identität: Die Angehörigen der Verwaltung haben in den meisten Ländern eine starke sektorale, fachliche Identität. Man fühlt sich einem Kreis von Fachkollegen und -kolleginnen zugehörig, mit denen man vielleicht studiert hat, mit denen man sich austauscht, die man bei Weiterbildungsveranstaltungen trifft. Vom Fachministerium erhofft man sich die Beförderung, die Versetzung von der Peripherie ins Zentrum. Dezentralisierung bedeutet für Regierungsbedienstete tendenziell eine Abkoppelung vom vertrauten fachlichen Bezugssystem und eine Unterordnung unter die tendenziell fachlich inkompetente Kontrolle der Lokalpolitiker. Das Bestreben nach Bewahrung sektoraler Identität und Zuordnung ist deshalb ein nicht zu unterschätzender beharrender Faktor zugunsten einer zentralistisch, sektoral-vertikal organisierten Verwaltung.

(3) Die Wahrnehmung der Realität in institutionellen Systemen tendiert dazu, Vorurteile zu bestätigen und zu verfestigen, welche der Festigung der eigenen Position dienen und eine Veränderung als riskant und verfrüht erscheinen lassen. «Helfer» sehen stets den fortbestehenden Unterstützungsbedarf und halten es stets für verfrüht, ihre Unterstützungsempfänger in die Eigenständigkeit zu entlassen. Zentralregierungen finden immer wieder heraus, dass die Distrikte oder Gemeinden «noch nicht» so weit sind, Aufgaben und vor allem Finanzen überantwortet zu bekommen und lassen sich diese Sichtweise durch von ihnen in Auftrag gegebene Gutachten gerne bestätigen. BEIER (1995) spricht in diesem Zusammenhang von «selbstre- ferentiellen Systemen» und belegt deren Existenz am Beispiel Indonesiens.

Der politische Wille zur Dezentralisierung wird aber auch massgeblich von Faktoren und Kräften außerhalb des politisch-institutionellen Systems beeinflusst. Die weit zurückreichenden historischen Grundlagen für das stark dezentrale föderalistische Regierungssystem in Deutschland sind ein eindrucksvoller Beleg hierfür. Zu diesen Faktoren gehören:

(1) Die relative Stärke regionaler Eliten und Fraktionen, welche dezentrale Kontrolle wollen und dafür eine Legitimationsbasis bei ihren regionalen Bevölkerungsgruppen haben. Wo der Wille, nach mehr Macht zu streben, fehlt, wird Macht nicht abgegeben werden.

(2) Die regionale Verteilung von Ressourcen beeinflusst die Möglichkeit zentraler bzw. dezentraler Kontrolle. Wenn die ökonomische Basis der Staatseinnahmen regional stark konzentriert ist (wie z.B. in Erdölstaaten), ist zentralistische Herrschaft leichter durchsetzbar als in Ländern, in denen die Wirtschaftskraft regional gleichmässig verteilt ist und Regionalregierungen weitgehend aus eigenen Einnahmequellen schöpfen können.

(3) Kulturelle und naturräumliche Diversität fördern häufig den Willen zu stärkerer regionaler Eigenständigkeit.

Es gibt also Länder mit günstigen Voraussetzungen für Dezentralisierung und solche, in denen sowohl die machtpolitische als auch die Ressourcenbasis hierfür schwach ausgeprägt ist. Im letztgenannten Fall ist es zweifelhaft, ob allein durch Überzeugungsarbeit bezüglich der Vorteilhaftigkeit dezentraler Regulierung eine Dezentralisierungspolitik Durchsetzungsund Erfolgschancen erhält.

\section{Intendierte positive und befürchtete negative Wirkungen dezentraler Regulierung}

Die erwarteten Vorteile wie auch die befürchteten Nachteile von Dezentralisierung sind weitgehend bekannt und wurden in der einschlägigen Literatur häufig zitiert (vgl. RondinELLı et al. 1989).

Sie werden deshalb hier ohne weitergehende Erörterung nur kurz zusammengefasst (vgl. Abb. 2). Diese Wirkungen stellen sich jedoch nicht automatisch ein, sondern nur dort, wo bestimmte weitere Bedingungen gegeben sind oder im Rahmen des Dezentralisierungsprozesses hergestellt werden. Auch der Vorteil von Dezentralisierung hängt also von historisch spezi- 
fischen gesellschaftlichen Gegebenheiten eines Landes ab. Diese Erfolgsbedingungen werden im Folgenden im Zusammenhang mit den Zielen bzw. erhofften positiven Wirkungen dargestellt.

(1) Dezentrale, kontextspezifische Entscheidungen vor Ort führen tendenziell zu situationsgerechteren und deshalb wirksameren Problemlösungen und zur besseren Nutzung lokaler Ressourcen. Dies gilt aber nur für Entscheidungsbereiche, für welche es relevantes lokales Wissen gibt. Außerdem ist es wichtig, bei der Ausgestaltung dezentraler Systeme dafür Sorge zu tragen, dass der Zugang zu professionellem Spezialwissen erhalten bleibt.

(2) Dezentrale Entscheidungsfindung ist eine wichtige Voraussetzung für Bürgerbeteiligung an Entscheidungsprozessen. Je näher an den davon Betroffenen die Entscheidungen gefällt werden, umso einfacher ist es, diesen unmittelbare Einflussmöglichkeiten zu eröffnen. Dezentralisierung impliziert aber nicht automatisch ein Mehr an Partizipation. In manchen Ländern sind lokale politische Strukturen weitaus weniger demokratisch und wesentlich autoritärer als die nationale Regierung (z.B. dort, wo auf dezentraler Ebene noch feudalismusähnliche Herrschaftsformen vorherrschen). Dezentralisierung eröffnet zwar Chancen für lokale Demokratie (STOckMAYER 1999), ist aber nicht mit Demokratisierung gleichzusetzen. Vielmehr ist die Existenz demokratischer Strukturen neben der - zu unterstützenden - Institutionalisierung partizipativer Planungsprozesse eine entscheidende Voraussetzung dafür, dass dezentralisierte Staatsmacht Partizipation tatsächlich stärkt.

(3) Ähnliches gilt für den Zusammenhang zwischen Dezentralisierung und Transparenz sowie Rechenschaftsablegung des Regierungshandelns. Je grösser die Bürgernähe der Entscheidungen, umso leichter ist es Kontrolle von unten herzustellen und Verantwortlichkeiten konkret festzumachen, um auf dieser Basis Anreize und Sanktionen (etwa durch Abwahl) für entwicklungs- und bürgerorientiertes Regierungshandeln zu schaffen.

Es ist jedoch eine verbreitete Fehleinschätzung, dass dezentralere Entscheidungen automatisch zu mehr Transparenz und Verantwortlichkeit gegenüber der Bevölkerung führen. In ländlichen Regionen ohne funktionierende Medien ist auch eine Distriktregierung, welche für mehrere hunderttausend Bewohner und Bewohnerinnen zuständig ist, für die Menschen weit weg. Wo die Beziehungen zwischen Regierenden bzw. lokalen Eliten und Regierten, d.h. Wählern und Wählerinnen kliente- lischer Natur sind, wird die Legitimation von gewählten Gemeindevätern durch Verteilung von Geschenken, nicht aber durch die Qualität und Entwicklungswirkung der Regierungsführung hergestellt. Nur dort, wo Klientelismus und Patronagebeziehungen keine zentrale Rolle mehr spielen, und wo zudem Mechanismen zur Stärkung von Transparenz und Rechenschaftsablegung gezielt gefördert werden, ist es wahrscheinlich, dass Dezentralisierung zu besserer Kontrolle von unten führt. Wichtig ist in diesem Zusammenhang auch, dass ein nennenswerter Teil der Finanzmittel der lokalen/regionalen Regierung aus lokalen Einnahmequellen kommt. Wo über eigenes Geld entschieden wird, sind die Bürger in der Regel wachsamer als dort, wo fremdes Geld ausgegeben wird.

(4) Dezentrale Regulierung kann zu wesentlichen Effizienzgewinnen durch Verkürzung der Instanzenwege und durch höhere Flexibilität des Verwaltungshandels führen. Solche potentiellen Effizienzvorteile lassen sich aber nur dort realisieren, wo genügend qualifiziertes Personal für die vielen regionalen bzw. lokalen Verwaltungen zur Verfügung steht. Bei der Ausgestaltung dezentralisierter Systeme ist darauf $\mathrm{zu}$ achten, dass durch Schaffung hinreichender Gemeindegrößen und durch eine Aufgabenzuordnung, die den verfügbaren personellen Ressourcen Rechnung trägt, tatsächlich leistungsfähige dezentrale Ver waltungseinheiten geschaffen werden.

(5) Dezentralisierung ist eine Voraussetzung für sektorübergreifende Koordination von Investitionen und Dienstleistungen auf regionaler bzw. lokaler Ebene. Nur dort, wo Sektorbehörden gezwungen sind, sich dem Koordinationsmandat einer dezentralen Regierung zu unterwerfen und wo sie die erforderliche Entscheidungsautonomie auf der dezentralen Ebene haben, ist ein zeitlich und räumlich koordiniertes Handeln verschiedener Träger öffentlicher Dienstleistungen möglich.

Dezentrale Regierung kann auch unerwünschte Wirkungen hervorrufen (vgl. Abb. 2a):

- Regionale Ungleichentwicklung kann verstärkt werden, wenn reiche Regionen ihren Reichtum und arme Regionen ihren Mangel selbst verwalten. Finanzausgleich und nationale Minimumstandards bei öffentlichen Leistungen sind Instrumente, die der Tendenz zur Verschärfung regionaler Disparitäten Grenzen setzen.

- Ein hohes Maß an regionaler bzw. lokaler Autonomie kann Tendenzen zur Desintegration des 
Nationalstaates bis hin zur Abspaltung Vorschub leisten. Unterschiedliche Bildungs- und Versorgungssysteme können zu Mobilitätshemmnissen werden. Konkurrenz zwischen regionalen Gebietskörperschaften um zentralörtliche Einrichtungen und Investitionen kann destruktiven Charakter annehmen, z.B. durch Duplizierung von (letztendlich dann unterausgelasteten) Einrichtungen oder durch eineinander Niederkonkurrieren im Bereich von Steuervorteilen zu Lasten der öffentlichen Kassen. Institutionalisierte Mechanismen horizontaler Koordination zwischen lokalen/regionalen Regierungen kann derartige Desintegrationstendenzen in akzeptablen Grenzen halten.

- Finanzministerien befürchten einen Verlust fiskalischer Stabilität, wenn eine Vielzahl lokaler Gebietskörperschaften autonom über Kreditaufnahme und Ausgabenvolumen entscheiden können. Nur durch zentrale Finanzkontrolle kann eine strikte Orientierung an makroökonomischen Stabilisierungszielen durchgesetzt werden.

- Dezentralisierung kann auch zu einer ineffizienten Aufblähung der Bürokratie und zur Duplizierung von Verwaltungseinrichtungen führen, wenn zusätzliche dezentrale Einrichtungen geschaffen werden, anstatt vorhandene Einrichtungen und Ressourcen einer dezentralen Kontrolle zu überantworten.

- Dezentralisierte Ressourcenkontrolle kann dazu führen, dass die Aneignungsmöglichkeiten lokaler Eliten erhöht werden und dadurch die Chancen des Zugangs benachteiligter Bevölkerungsgruppen $\mathrm{zu}$ öffentlichen Leistungen tendenziell noch weiter verringert werden. Diese - häufig beobachteten Tendenzen können nur verhindert werden, wenn Dezentralisierung mit Schritten der Demokratisierung und der Stärkung von Möglichkeiten der Bürgerbeteiligung einhergeht.

Dezentralisierung an sich ist noch kein Beitrag zu Entwicklung und zu nachhaltiger Armutsminderung. Dezentralisierte Ressourcenkontrolle kann unter bestimmten gesellschaftlichen Bedingungen Tendenzen zur Unterentwicklung, Verarmung, Ressourcenzerstörung und zu gewaltsam ausgetragenen Konflikten verschärfen.

Dezentralisierung an sich schafft auch nicht mehr Demokratie. Dezentralisierung erfordert ein gewisses $\mathrm{Ma}$ an Demokratie um erfolgreich zu sein. Und sie erfordert ein an den Bedingungen des jeweiligen Landes orientiertes, fein ausgeklügeltes System der Verteilung von Aufgaben auf verschiedenen Regierungsebenen und der wechselseitigen Kontrollen von unten und von oben.
Dezentralisierung bedarf einer landesspezifischen und am jeweiligen gesellschaftlichen, historischen Kontext orientierten Ausgestaltung. Es gibt kein Patentrezept. Dies lässt sich am Fall Südafrika gut illustrieren.

\section{Südafrika: Voraussetzungen für Dezentralisierung nach der Apartheid}

Die historischen und räumlichen Bedingungen für ein dezentrales politisches und administratives System waren in Südafrika am Ende der Apartheid-Ära 1994 überwiegend günstig: Die Größe und naturräumliche Heterogenität des Landes in Verbindung mit einer Vielfalt an auf die Regionen des Landes verteilten Ressourcen begünstigen eine Regionalisierung ebenso wie die soziokulturelle Diversität der multi-rassischen und multi-ethnischen südafrikanischen Gesellschaft. Die räumlich stark konzentrierten und zentralistisch kontrollierbaren Ressourcen (insbesondere Gold) verlieren demgegenüber an Bedeutung. Der politische Umbruch zu Beginn der neunziger Jahre, welcher ohnehin eine radikale Umgestaltung des politischinstitutionellen Systems erforderte, bot Chancen für den Aufbau eines dezentralen Systems, die sich andernorts aufgrund der Beharrungskräfte fest etablierter Machtstrukturen nur selten ergeben. Es gab 1994 in Südafrika also ein «offenes Fenster» für Dezentralisierung, es gab Kräfte, die diese wollten, und es gab Ressourcen, die eine tragfähige Basis für eine relativ weitgehende regionale Autonomie boten.

Eben diese begünstigenden Faktoren stellten aber auch ein Bedrohungspotential für die angestrebte Einheit des Landes, für eine Integration der südafrikanischen Gesellschaft und für die politisch gewünschte Angleichung der Lebensbedingungen dar. Insbesondere bei den regionalen Eliten in Kwazulu-Natal und in der Westkap Region (am Kap) gibt es separatistische Tendenzen. Die beiden wichtigsten Oppositionsparteien zur nationalen ANC (African National Congress) Regierung haben in diesen Regionen ihre Wählerbasis und haben nur dort gute Chancen für eine regierungsfähige Mehrheit. Sie stellten folglich die dezentralisierungsfreundlichen Fraktionen. Auch auf kleinräumiger Ebene gibt es starke Befürworter einer Dezentralisierung im Sinne einer weitgehenden kommunalen Selbstverwaltung: die Bewohner und Bewohnerinnen reicher weißer Gemeinden bzw. Gemeindeteile haben ein Interesse, unter sich zu bleiben, um ihren Wohlstand wenigstens auf kommunaler Ebene nicht mit den armen und infrastrukturell vernachlässigten Regionen teilen zu müssen. Eine sehr weitgehende Dezentralisierung käme letztlich einer Fortsetzung der Apartheidpolitik der "getrennten Entwicklung» gleich. 
Die beiden vorrangigen politischen Ziele nach Ende der Apartheid, die Integration der südafrikanischen Gesellschaft und die Angleichung der extrem ungleichen Lebensbedingungen durch Umverteilung gehören (vgl. Abschnitt 4) nun gerade nicht zu den Vorteilen dezentralistischer Regulierung. Dasselbe gilt für das zentrale wirtschaftspolitische Ziel der neuen südafrikanischen Regierung, durch fiskalische Stabilität eine Basis für Investitionen und internationale Wettbewerbsfähigkeit zu schaffen. Aus diesen Gründen, aber auch aufgrund seiner zentralistischen Tradition als Befreiungskampforganisation, neigt die Mehrheit im ANC eher zu zentralistischen Positionen. Die Überwindung der Strukturen der Apartheid erfordert politisch-administrative Einheiten, welche groß genug sind, um das Ungleiche zusammenzubringen. Das gilt bei großräumiger Betrachtung für die Reintegration der "Homelands» ebenso wie bei der kleinräumigen Betrachtung für die Integration schwarzer und weißer Wohngebiete im Rahmen einer Gemeindegebietsreform.

\section{Die politischen und verfassungsrechtlichen Rahmenbedingungen im «neuen» Südafrika}

Angesichts der dargestellten Bedingungen, Interessen und Ziele galt es im Rahmen der Diskussion um eine neue Verfassung einen Kompromiss zwischen zentralistischen und dezentralistischen Kräften zu finden. Zwar konnte man sich nicht auf das von deutschen Beratern propagierte föderalistische Modell einigen. Die damit verbundene Eigenständigkeit der Provinzen ging dem ANC zu weit. Immerhin kam es zu dem Modell dreier eigener («distinct», nicht aber «independent»!), aber miteinander kooperierender Regierungssphären (nicht -ebenen) mit gewählten Parlamenten und Regierungen auf nationaler, Provinz- und Gemeindeebene. Die Verfassung sieht zahlreiche Eingriffsrechte der nationalen Sphäre in die Belange der anderen beiden Sphären vor. Die Regelung der Verteilung der Zuständigkeit ist in weiten Kompetenzbereichen uneindeutig. Der Verfassungskompromiss lässt weiten Raum für Interpretationen und für Entwicklungen. Das Bemerkenswerte am südafrikanischen «Drei Sphären-Modell» ist nicht so sehr, dass es weniger hierarchisch klingt als ein «Drei Ebenen-Modell», sondern, dass die drei einander überlappenden Sphären explizit direkte Beziehungen zwischen nationaler und kommunaler Sphäre unter Umgehung der Provinzen zulassen (vgl. Abb. 3).

Seit der Verabschiedung der Verfassung unternahm die neue Regierung eine Reihe von Anstrengungen zur Transformation und Stärkung der Gemeinden:

- 1995 wurden rassisch gemischte Übergangsgemeinden gegründet und entsprechende Gemeinderäte gewählt. Dies erfolgte nach dem Prinzip schwarze Mehrheit (im Gemeinderat) mit «weißer» Verwaltungserfahrung und «weißen» Steuereinnahmen zusammenzubringen. Dies war ein erster Schritt in Richtung Integration und Umverteilung, welcher aber noch nicht zu überlebensfähigen oder gar entwicklungsfähigen Gemeinden führte. Viele Übergangsgemeinden waren nicht mehr als Wahlbezirke ohne jegliche administrative Strukturen.

- Ende 1996 wurde per Gesetz verordnet, dass alle Gemeinden «Integrierte Entwicklungspläne» erstellen sollten. Der angestrebte gemeinsame Planungsprozess sollte zu einer Ausrichtung aller Gruppen in den Gemeinden hin auf gemeinsame Visionen und Entwicklungsziele führen und somit zu einem zentralen Integrationsinstrument werden.

- Im «White Paper on Local Government» von 1998, welches als politischer Orientierungsrahmen für die Sphäre der Kommunalregierungen dient, wird den Gemeinden als der Regierungssphäre die am nächsten am Bürger dran ist, eine entscheidende Rolle als Träger von Entwicklungsprozessen zugeschrieben. Gemeinden sollen nicht nur die Hauptverantwortung bei der Bereitstellung

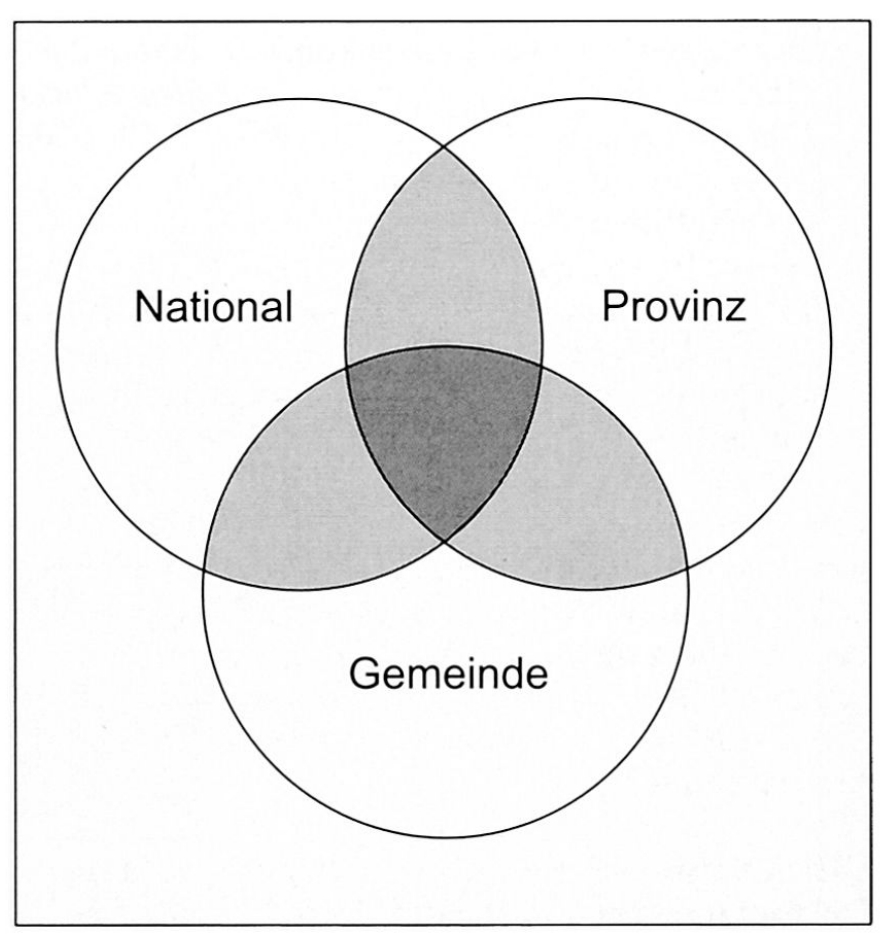

Abb. 3: Die drei Regierungssphären in Südafrika The three spheres of government in South Africa Les trois sphères gouvernementales en Afrique du Sud Entwurf: T. RAUCH 
öffentlicher Leistungen tragen, sondern auch die lokale Wirtschaftsentwicklung fördern, die Integration der durch Apartheid getrennten Siedlungsgebiete und Bevölkerungsgruppen vorantreiben und eine Angleichung der Lebensbedingungen im Gemeindegebiet bewirken (MINISTRY FOR Provincial Affairs and Constitutional Development 1998: 17ff.). Der durch das «White Paper» gesetzte Rahmen wurde 1999 und 2000 in Gesetzesform gegossen.

- 1999 wurde eine Gemeindegebietsreform beschlossen mit dem Ziel, größere überlebens- und umverteilungsfähige Gemeinden zu schaffen. Die Zahl der Gemeinden wurde von 847 auf 284 reduziert. Die durchschnittliche Gemeindegröße stieg damit auf über 100'000 Einwohner. Im Dezember 2000 wurde mit den Gemeinderatswahlen die Gebietsreform und damit die Phase der Übergangsgemeinden abgeschlossen. Der administrative Reorganisationsprozess ist aber noch in vollem Gange.

Ein entscheidender noch ausstehender Schritt hin zur entwicklungsorientierten Selbstverwaltungsgemeinde ist die Gemeindefinanzreform. Nur wirtschaftsstarke und großstädtische Gemeinden können aus eigenen kommunalen Einnahmequellen einen wesentlichen Teil ihrer Ausgaben bestreiten. Kleinere und ländliche Gemeinden benötigen hierzu einen höheren Anteil an den Steuerquellen der nationalen Regierung. Mit der Gemeindegebietsreform wurde eine entscheidende Voraussetzung für eine stärkere fiskalische Dezentralisierung geschaffen. Nun muß sich zeigen, ob der politische Wille stark genug ist, den Gemeinden nicht nur anspruchsvolle Aufgaben, sondern auch die zu ihrer Erfüllung erforderlichen Ressourcen anzuvertrauen.

\section{Die Herausforderung: Die schwierigsten Aufgaben für den schwächsten Träger?}

Die sich neu konstituierenden Gemeinden mit überwiegend unerfahrenen Gemeinderäten und mit Verwaltungen, die z. T. an den Verfahrensweisen der eher kontroll- als entwicklungsorientierten Apartheidverwaltungen festhalten, stehen schier unlösbar erscheinenden Aufgaben gegenüber (vgl. Abb. 4). Beispielsweise geht es dabei darum, mit eng begrenzten

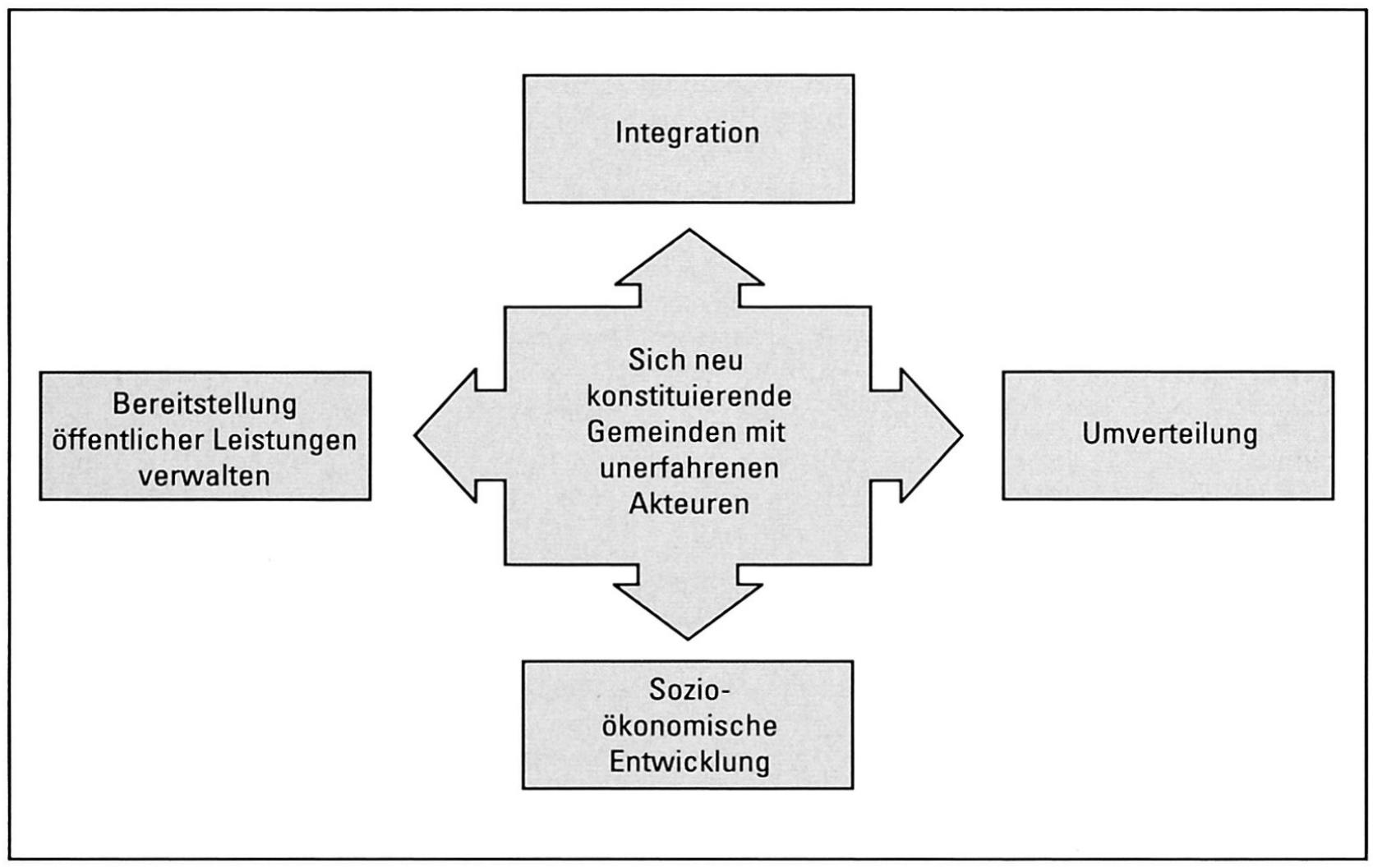

Abb. 4: «Entwicklungsorientierte Gemeinden»: Die Herausforderung

The Challenge: Development-oriented Local Government

Développement communal: l'enjeu

Entwurf:T. RAUCH 
finanziellen Mitteln eine Basisinfrastruktur für ca. $80 \%$ der bislang vernachlässigten nicht-weißen Bevölkerung bereitstellen, ohne die den Standards hochentwickelter Industriegesellschaften entsprechende Infrastruktur in den bislang weißen Gemeindeteilen verfallen zu lassen. Die Größe der Herausforderung lässt sich an einem Vergleich mit der Umverteilungsproblematik im wiedervereinigten Deutschland illustrieren: dort ging es um eine Umverteilung von $80 \%$ der Bevölkerung im reicheren Landesteil auf $20 \%$ im ärmeren Landesteil. In südafrikanischen Gemeinden soll von $20 \% \mathrm{im}$ reicheren Gemeindeteil auf $80 \%$ der Bevölkerung in den ärmeren Gemeindeteilen umverteilt werden.

Ähnlich verhält es sich mit der Problematik der Integration. Konnte im wiedervereinigten Deutschland noch das Motto von Willy BRANDT gelten: «Zusammenfügen was zusammen gehört», so besteht die Herausforderung für Südafrika eher darin, zusammenzufügen, was immer getrennt gehalten wurde und einander bekämpft hat.

Das Konfliktszenario auf lokaler Ebene in Südafrika lässt sich aber nicht auf den Schwarz-Weiß-, oder ArmReich-Konflikt reduzieren. Neu gewählte Gemeinderäte stehen in Konkurrenz um Einfluss gegenüber außerparlamentarischen Organisationsformen, die in den letzten Jahren der Apartheid-Ära bestimmend für lokal-politische Entscheidungen waren. In ländlichen Gebieten konkurrieren sie mit traditionellen Herrschaftsstrukturen um lokal-politische Zuständigkeiten. Derartige Konflikte resultieren häufig in Entscheidungsblockaden und entsprechenden Verzögerungen bei der Durchführung dringender Entwicklungsmaßnahmen. Wo die Gemeinderegierungen nicht zu Entscheidungen und Aktion fähig sind, wo budgetierte Mittel nicht abfließen, versuchen die staatlichen Ministerien und die Bevölkerung sich unter Umgehung der Kommunen miteinander kurz zu schließen. Ohne die erforderliche Planung vor Ort führt dies oft zu völlig unangepassten Fehlinvestitionen und somit zur Verschwendung knapper Ressourcen.

Alle Beteiligten in Südafrika sind sich darüber einig, dass die Mehrzahl der Gemeinden für die ihnen zugemuteten Aufgaben nicht gerüstet sind und massiver Unterstützung bedürfen. In der Einführung geeigneter kommunaler Planungs-, d.h. Entscheidungsverfahren wird ein entscheidendes Instrument zur Bewältigung der neuen Herausforderung gesehen.

\section{8 Überwindung von Apartheid durch dezentrale Entwicklungsplanung}

Integrierte Entwicklungsplanung wird vom zuständigen Ministerium der südafrikanischen Regierung verstanden als ein konsultativer, strategischer und umsetzungsorientierter Entscheidungsprozess über die Prioritäten, Visionen, Ziele und Entwicklungsmaßnahmen im Gemeindegebiet. An den «integrierten Entwicklungsplänen» sollen sich die Budgets der Gemeinden, aber auch die Planungen der Sektorministerien orientieren. Die Pläne sollen als Grundlage für Landnutzungsmanagement und als Orientierung für die Entscheidungen öffentlicher und privater Unternehmen dienen. Sozialräumliche Integration und Angleichung der Lebensbedingungen insbesondere im Bereich des Zugangs zu grundbedarfsorientierten öffentlichen Leistungen und Einrichtungen gehören zu den ausdrücklichen Zielen, an denen sich «Integrierte Entwicklungspläne» orientieren sollen.

Diese Form von «Integrierter Entwicklungsplanung» befindet sich noch in ihrem Anfangsstadium. Noch besteht die Planungsrealität in den meisten Übergangsgemeinden in der Erstellung umfangreicher und wenig strategischer und umsetzungsorientierter Planungsdokumente durch private Planungsbüros. Dennoch gibt es bereits zahlreiche «best practice»-Beispiele, die zeigen, wie kommunale Entwicklungsplanung dazu beitragen kann, Integrationsprozesse in die Wege zu leiten und Umverteilungsspielräume zu eröffnen:

- Das Planungsverfahren half in vielen Fällen Konflikte und daraus resultierende Entscheidungsblokkaden zu überwinden, indem divergierende Interessen mit Hilfe transparenter Priorisierungsverfahren zum Ausgleich gebracht werden. Als wichtig hierfür erwies sich der mittelfristige (5-jährige) Planungszeitrahmen. Minderheitenbedürfnisse mussten nicht völlig verworfen werden, sondern kommen als nachgeordnete Prioritäten später zum Zuge oder erhielten einen geringeren Anteil der Budgetmittel. Die Frage von "Gewinnern» und «Verlierern» des Planungsprozesses stellt sich damit in einer weniger dramatischen Weise. Kompromisse werden dadurch leichter. Entscheidungen kommen rechtzeitig für die Budgetplanung zustande. Den Erwartungen der Bevölkerung nach schnelleren Verbesserungen kann leichter entsprochen werden.

- Die Einigung auf ein konsultatives, systematisches und transparentes Planungsverfahren fördert die Akzeptanz der Planungsergebnisse bei allen Beteiligten und erleichtert somit den Umverteilungsprozess. Für eine bislang privilegierte weiße Minderheit ist es leichter zu ertragen, dass ein großer Teil der von ihren erwirtschafteten Steuern und Abgaben zugunsten der Verbesserung der Situation in schwarzen Siedlungsgebieten ausgegeben wird, wenn dies nicht einfach von der Gemeinderatsmehrheit über ihre Köpfe hinweg beschlossen wird, sondern Ergebnis sorgfältiger Analyse, ratio- 
naler, zielorientierter Diskurse und fairer Interessensabwägung ist. Einer schwarzen Mehrheit fällt es als Ergebnis eines solchen gemeinsamen Prozesses meist leichter zu akzeptieren, dass auch die Instandhaltung der Infrastruktur in den weißen Siedlungsgebieten ein erstrebenswertes Ziel ist, für das ein Teil der Mittel abzuzweigen ist.

- Die Einbeziehung von Vertretern und Vertreterinnen aller Bevölkerungsgruppen in einen gemeinsamen Planungs- und Entscheidungsprozess, die gemeinsame (wenngleich kontroverse) Auseinandersetzung um Ziele und Mittel fördert den Integrationsprozess der verschiedenen Bevölkerungsgruppen. Nicht wenige weiße Gemeindebewohner lernen im Rahmen des Planungsprozesses erstmals die Situation in den schwarzen Siedlungsgebieten kennen.

- Ein Leitmotiv kommunaler Entwicklungsplanung in Südafrika ist die Suche nach «common ground», d.h. nach gemeinsamen Interessen, nach sogenannten «win-win»-Lösungen. Man ist bestrebt, durch einen Einigungsprozess auf eine gemeinsame Vision für die Gemeinde, auf gemeinsame Ziele und Strategien, sowie auf Maßnahmen, die den Interessen der Bevölkerungsgruppen entgegenkommen, Verteilungskonflikte zu entschärfen. Kommunale Wirtschaftsförderungsstrategien sollen Investitionsmöglichkeiten für kapitalkräftige Bevölkerungsgruppen und gleichzeitig Arbeitsplätze für die Arbeitslosen schaffen. Das Image oder die Standortattraktivität einer Stadt sollen zu Gunsten aller Bewohner und Bewohnerinnen aufpoliert werden. Wie weit und wie tragfähig der «common ground» im jeweiligen Fall tatsächlich ist, bedarf sicherlich einer kritischen Analyse. Wichtig ist zunächst, dass der Prozess der Suche danach die Gemeinsamkeit in den Vordergrund und das Trennende in den Hintergrund rückt.

- Strategisch planen heißt u.a. Suche nach Mitteln und Wegen zur effektiveren Verwendung knapper Ressourcen durch angemessenere, kostengünstigere Problemlösungen. Dort, wo man sich beispielsweise dafür entscheidet, die unterausgelastete Sekundarschule im weißen Gemeindeteil durch ein Schulbussystem für schwarze Schüler und Schülerinnen zugänglich zu machen, statt eine zweite schlecht ausgelastete Schule im schwarzen Siedlungsgebiet zu bauen, werden Mittel etwa für die Verbesserung der Gesundheitsversorgung in den bislang vernachlässigten Gebieten frei. Dort, wo man sich dazu durchringt auf Wasserklosetts zugunsten von Latrinen zu verzichten, kann die Zahl derer, die in den Genuss einer verbesserten Trinkwasserversorgung kommen, in gegebener Zeit verdoppelt werden. Umverteilungsspielräume werden durch derartige intelligente Lösungen erweitert, Verteilungskonflikte entschärft.

Bislang stellen derartige Beispiele nicht die Regel sondern die Ausnahme dar. Sie zeigen jedoch die Potentiale einer konsultativen und strategischen kommunalen Entwicklungsplanung für die Überwindung der Strukturen der Apartheid auf lokaler Ebene auf (vgl. DPLG 2001).

Wo, wie in Südafrika, Segregation und soziale Gegensätze in enger Nachbarschaft, in kleinräumigem Maßstab vorzufinden sind, bedarf es dezentraler, lokaler Aushandlungs- und Einigungsprozesse, um zu einer Integration und einer Angleichung der Lebensbedingungen zu gelangen. Zentralstaatliche Steuer- und Sozialsysteme und arbeitsmarktpolitische Instrumente mögen das Ihre zum Abbau von Diskriminierung und Ausgrenzung nicht-weißer Bevölkerungsgruppen beitragen. Sie können aber die Rolle der rassisch gemischten Gemeinden als Forum für die Aushandlung von einvernehmlichen, den gegensätzlichen Interessen Rechnung tragenden Entwicklungspfaden nicht ersetzen.

\section{Die Schwierigkeiten und Unzulänglichkeiten dezentraler kommunaler Entwicklungsplanung und Ansätze zu deren Überwindung}

Die Zahl der Fälle, bei welchen integrierte Entwicklungsplanung auf kommunaler Ebene ihre Eignung als Instrument zur Überwindung von Apartheid bereits bewiesen hat, ist noch gering. Dies liegt daran, daß die bisherigen Übergangsgemeinden meistens noch nicht für die Organisation solch eines konsultativen und strategischen Planungsprozesses gerüstet waren und in ihrer Hilflosigkeit die Aufgabe auf Planungsbüros ausgelagert hatten, welche ihre Rolle in alter Tradition darin sahen, Planungsdokumente am Schreibtisch anzufertigen. Es waren aber nicht alleine diese Geburtswehen und Kinderkrankheiten, die die Einführung dezentraler Entwicklungsplanung erschwerten. Manche Schwierigkeiten und Unzulänglichkeiten im Planungsprozess waren ein Resultat unzureichend beantworteter Grundsatzfragen der Ausgestaltung dezentraler Planungssysteme. (Der Verfasser dieses Beitrags arbeitet als Berater für die GTZ (Gesellschaft für Technische Zusammenarbeit) an der Konzipierung des neuen kommunalen Planungsverfahrens mit. Die Aussagen reflektieren persönliche Einschätzungen basierend auf den Erfahrungen aus der Teilnahme an diesem Prozess.)

(1) Die Frage der angemessenen Gemeindegröße: Viele der südafrikanischen Gemeinden waren (mit 
weniger als 5'000 Einwohnern) nicht nur zu klein, um sich eine leistungsfähige Gemeindeverwaltung leisten zu können. Sie waren auch zu klein, um die angestrebten Integrations- und Umverteilungsprozesse zu ermöglichen. Die Zusammenführung schwarzer und weißer, armer und reicher Siedlungsgebiete erfordert relativ große Gemeindegebiete. Je größer aber die Gemeindegebiete und die Bevölkerungszahl, um so schwieriger ist es Bürgernähe zu der Gemeindeverwaltung herzustellen. Die südafrikanische Regierung entschied sich in diesem Konflikt zugunsten leistungs-, umverteilungs- und integrationsfähiger Großgemeinden. Die Zahl der Gemeinden wurde im Rahmen der Gemeindegebietsreform im Jahr 2000 von 847 auf 284 reduziert. Die durchschnittliche Gemeindegröße liegt damit bei deutlich über 100'000 Einwohnern. Der in der intendierten starken Rolle der Gemeinden zum Ausdruck kommende Dezentralisierungswille wird also durch relativ zentralistisch organisierte Großgemeinden (und auch durch eine Stärkung der Distrikte als zweite Ebene im Rahmen von «local government») relativiert.

(2) Integrierte Entwicklungsplanung erfordert fachliche Kapazitäten, die auch in den meisten der vergrößerten Gemeinden nicht vorhanden sind und die auf dieser Ebene auch nicht auf ökonomisch tragfähiger Basis geschaffen werden können. Für viele ärmere Gemeinden ist ein eigenes kommunales Planungsamt zu teuer. Folglich tendieren die meisten Gemeinden dazu, Planungsleistungen bei kommerziellen Planungsbüros einzukaufen, statt in der Gemeinde einen konsultativen und strategischen Entwicklungsprozess zu organisieren. Die Lösung des Dilemmas zwischen der anspruchsvollen Aufgabe strategischer und integrierter Entwicklungsplanung und den begrenzten Kapazitäten vieler Gemeinden wurde in gemeindeübergreifenden Planungsberatungszentren auf Distriktebene gesehen. Ein solches Zentrum unterstützt die Planungsprozesse in etwa fünf Gemeinden. Die Managementverantwortung für den Planungsprozess bleibt bei den Gemeinden. Planungsbüros werden gezielt für Spezialaufgaben miteinbezogen. Dezentrale Planungsverantwortung wird somit mit zentraler Bereitstellung von fachlicher Planungsleistung kombiniert.

(3) Die Frage der Form der Bürgerbeteiligung wurde von der südafrikanischen Gemeindegesetzgebung weitgehend offengelassen (Republic of South Africa 2000: 10ff.). Ambitiösen Grundsätzen der Bürgergemeinde steht ein äußerst geringer Grad der Regulierung der Beteiligungsrechte der Bürger gegenüber. Es bleibt den Gemeinden selbst überlassen, geeignete Beteiligungsformen festzulegen. Begründet wird diese Unterregulierung der Beteiligungsmöglichkeiten mit der Unterschiedlichkeit der Partizipationskulturen der verschiedenen Bevölkerungsgruppen und in städtischen und ländlichen Gemeinden. Das vorgeschlagene Planungsverfahren versucht diese Gesetzeslücke auszufüllen, indem es mit der Einrichtung von repräsentativen Foren (in welchen alle Siedlungsgebiete und alle sozio-ökonomischen Gruppen vertreten sind) bestimmte Minimumvoraussetzungen für Bürgerbeteiligung institutionalisiert ohne damit weitergehende Beteiligungsprozesse vorherzubestimmen oder zu verhindern. Dezentralisierung von Entscheidungsmacht darf nicht so weit gehen, daß Bürger in verschiedenen Landesteilen völlig unterschiedliche demokratische Rechte innehaben.

(4) Die Frage der fiskalischen Dezentralisierung ist in Südafrika noch unzureichend gelöst. Die kommunalen Einnahmequellen (Grund- und Gewerbesteuern sowie diverse Gebühren und Abgaben) sind für Gemeinden in wirtschaftsschwachen Regionen unzureichend. Die meisten Transferzahlungen seitens der nationalen Regierung sind zweckgebunden und konditioniert. Nur $1.5 \%$ der nationalen Einnahmequellen gehen als unkonditionierter Zuschuss («equitable share») an die Gemeinden. Dem Mandat zur Entwicklungsplanung, das den Gemeinden «großzügig» übertragen wurde, entspricht das ihnen zur Disposition überlassene finanzielle Mittelvolumen also in keiner Weise. Das ist angesichts des bisherigen Provisorismus der Übergangsgemeinden, in welchem vielerorts noch keine hinreichende Finanzkontrolle gewährleistet war, durchaus nachvollziehbar. Strategische Planung aber ist ohne freie Verfügungsgewalt über die vorgesehenen Finanzmittel nicht möglich. Strategische Planung basiert auf dem Prinzip, gegebene knappe Mittel so kostengünstig und effizient wie möglich zu verwenden. Planung zur Erlangung externer Mittel hingegen erfolgt nach dem Prinzip, für einen gegebenen Zweck das Doppelte des Erforderlichen zu veranschlagen, um möglichst viel zu bekommen ein Anreizsystem, das der Logik strategischer Planung also diametral entgegengesetzt ist. Da die Auseinandersetzung um eine stärkere fiskalische Dezentralisierung noch einige Jahre andauern wird, sieht das kommunale Planungsverfahren vor, mit einem kalkulatorischen Finanzrahmen zu planen, um so die Bedingungen für einen strategischen Suchprozess nach effizienten und effektiven Problemlösungen zu schaffen. 


\section{Fazit}

Das Beispiel Südafrika verdeutlicht auf eindrucksvolle Weise, dass es kein generelles Modell politisch-administrativer Dezentralisierung geben kann. Die Beantwortung der Frage nach dem angemessenen Grad und der richtigen Form dezentraler Regulierung hängt vielmehr von einer Vielzahl gesellschaftlicher Ziele und Bedingungen ab. In Südafrika kam es aufgrund des Einflusses regionaler Eliten zu einem relativ dezentralen Modell mit drei eigenständigen «Regierungssphären». Andererseits erforderten die starke gesellschaftliche Bedeutung von Integrations- und Umverteilungszielen sowie die begrenzten administrativen Kapazitäten eine relativ zentralistische Lösung (in Form von Großgemeinden) auf der lokalen Ebene.

Der Fall Südafrika zeigt aber auch, dass Zielsetzungen, wie Integration und Verteilungsgerechtigkeit, welche gemeinhin als typisch zentralstaatliche Aufgaben betrachtet werden, in bestimmten Konstellationen besser durch dezentrale Entscheidungsprozesse erreicht werden. Dies gilt beispielsweise dann, wenn es sich - wie bei den Folgen der Apartheid - um Integrations- und Angleichungsprozesse auf relativ kleinräumiger Maßstabsebene handelt. Hier bedarf es lokaler Aushandlungs- und Problemlösungsprozesse, um zu allseits akzeptablen Lösungen zu gelangen.

So unterschiedlich die angemessene Verteilung von Aufgaben, Macht und Ressourcen auf die verschiedenen Regierungs- und Verwaltungsebenen in verschieden Ländern auch sein mag, so gibt es doch eine Grundregel, an welcher sich Dezentralisierungspolitik überall orientieren sollte: Dezentralisierung von Funktionen sollte einhergehen mit Dezentralisierung finanzieller Mittel und mit Demokratisierung, d.h. zunehmender Bürgerbeteiligung. Nur wenn Gemeinden über finanzielle Mittel verfügen macht Bürgerbeteiligung an kommunalen Entscheidungen auf Dauer Sinn. Umgekehrt gilt: Nur wenn durch Bürgerbeteiligung Transparenz und Kontrolle von unten gewährleistet sind, kann sichergestellt werden, dass kommunale Verfügungsgewalt über öffentliche Finanzen der Gemeindebevölkerung wirklich zugute kommt.

\section{Literatur}

BEIER,C. (1995): Dezentralisierung und Entwicklungsmanagement in Indonesien. Beobachtungen zur politischadministrativen und zur wissenschaftlichen Kommunikation: ein systemtheoretischer Versuch. - Saarbrücken: Verlag für Entwicklungspolitik Breitenbach.

DPLG (Department of Provincial and Local Government) (2001): Integrated Development Planning Guide Pack. - Pretoria.

Elias, N. (1976): Über den Prozess der Zivilisation.
Soziogenetische und psychogenetische Untersuchungen. Band 2: Wandlungen der Gesellschaft. Entwurf zu einer Theorie der Zivilisation. - Frankfurt a.M.: Suhrkamp.

Hyden, G. (1985): No Shortcuts to Progress. African Development Management in Perspective. - London, Ibadan, Nairobi: Heinemann.

Ministry for Provincial Affairs and ConstitutioNal Development (ed.) (1998): White Paper on Local Government (WPLG). - Pretoria.

NorTh, D.C. (1992): Institutionen, institutioneller Wandel und Wirtschaftsleistung.-Tübingen:C.B.Mohr (Paul Siebeck).

RAuCH, T. (1986): Dezentralisierung, Bauern und Staat in Zambia. - In: Zeitschrift für Wirtschaftsgeographie 30/3-4: 52-71.

RAUCH, T. (1996): Ländliche Regionalentwicklung im Spannungsfeld zwischen Weltmarkt, Staatsmacht und kleinbäuerlichen Strategien. - Saarbrücken: Verlag für Entwicklungspolitik Breitenbach.

Republick of South Africa (2000): Municipal Systems Act 2000. - Pretoria.

Rondinelli, D.A., McCullough, J.S. \& R.W. Johnson (1989): Analysing Decentralization Policies in Developing Countries: A Political-Economy Framework. In: Development and Change 20:57-87.

StockmAYER, A. (1999): Decentralization: Global fad or recipe for sustainable local development? - In: Agriculture and Rural Development 6/1:3-6.

Zusammenfassung: Dezentralisierung ist kein Allheilmittel! - Zur Notwendigkeit einer kontextspezifischen Dezentralisierungspolitik am Beispiel der Kommunalentwicklung in Südafrika

Institutionelle Reformen werden als entscheidender Ansatzpunkt für erfolgreiche Entwicklungsprozesse betrachtet. Bezüglich der konkreten Ausgestaltung institutioneller Regulierung gibt es jedoch keine Patentrezepte. Vielmehr sind situationsgerechte institutionelle Reformen an den spezifischen sozialen, ökonomischen und physischen Bedingungen eines Landes bzw. einer Gesellschaft zu orientieren.

Dies gilt auch für Dezentralisierung als grundlegendem institutionellem Transformationsprozess. Es gibt weder ein idealtypisches Muster dezentraler Regulierung noch für erfolgreiche Dezentralisierungsprozesse. Die angemessene Verteilung von Aufgaben, Macht und Ressourcen auf verschiedene politische bzw. administrative Ebenen hängt vielmehr von den spezifischen historisch-geographischen Bedingungen ab. Dies wird im vorliegenden Beitrag am Beispiel Südafrikas illustriert, wo nach Ende des Apartheidsystems dezentrale Entwicklungsplanung dazu beitragen soll, Integration und Umverteilungsprozesse auf lokaler Ebene auf Basis demokratischer lokaler Aushandlungs- und Entscheidungsprozesse herbeizuführen. 
Summary: A Critical Look at Decentralisation - Communal development in South Africa as an example of the importance of a local-based decentralisation politics

Institutional reform is considered to be the key for successful development. However, for reform to be appropriate, the nature of institutional change needs to be closely related to the specific social, economic and physical conditions of a country and a society. This also applies to the fundamental institutional transformational process of decentralisation. There is neither an ideal pattern of, nor an ideal path towards a decentralised system of regulation. The appropriate distribution of functions, powers and resources between different levels of government depends on a range of historically and geographically specific conditions. This can be illustrated by the case of post-apartheid South Africa, where decentralised municipal development planning is expected to promote integration and redistribution processes at the local level by providing a local forum for democratic negotiation between different stakeholders.

Résumé: La décentralisation n'est pas une panacée! Nécessité d'une politique de décentralisation adaptée au contexte, l'exemple du développement communal en Afrique du Sud

Les réformes institutionnelles sons considérées comme étant le point de départ décisif des processus de développement réussis. Cependant, il n'a y pas de recette miracle pour trouver quelle forme concrète donner à une régulation institutionnelle. Pour qu'elles soient adaptées à la situation, les réformes institutionnelles doivent tenir compte des conditions sociales, économiques et physiques spécifiques d'un pays ou d'une société.
Ceci s'applique aussi à la décentralisation en tant que processus institutionnel fondamental de transformation. Il n'existe aucun modèle idéal de régulation décentralisée, ni aucun modèle garantissant la réussite des processus de décentralisation. Comme le montre l'exemple de l'Afrique du Sud, le partage équitable des tâches, du pouivoir et des ressources aux différents niveaux politiques ou administratifs dépend davantage des conditions historiques et géographiques spécifiques: dans ce pays, après la fin de l'apartheid, la planification décentralisée du développement devrait favoriser les processus d'intégration et de redistribution au niveau local en sa basant sur les processus démocratiques locaux de négociation et de décision.

Prof. Dr. Theo Rauch, c/o GTZ, P.O. Box 13732, Pretoria 0028, Südafrika.

e-mail:Vira@dso.pwv.gov.za

Manuskripteingang/received/rentrée du manuscrit: 12.2.2001

Annahme zum Druck/accepted for publication/ accepation à l'impression: 17.4.2001 